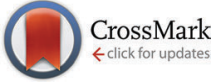

Cite this: Phys. Chem. Chem. Phys., $2016,18,11556$

DOI: $10.1039 / c 6 c p 90085 b$

www.rsc.org/pccp

\title{
Correction: Large-size nanosheets of 9,10-bis(phenylethynyl)anthracene with high photoresponse and light emission anisotropy
}

\author{
Juan-Ye Wang, ${ }^{\text {ab }}$ Hong-Dan Peng, ${ }^{b}$ Jia-Mei Yang, ${ }^{b}$ Jing-Hui Yan*a and \\ Ge-Bo Pan*b
}

Correction for 'Large-size nanosheets of 9,10-bis(phenylethynyl)anthracene with high photoresponse and light emission anisotropy' by Juan-Ye Wang et al., Phys. Chem. Chem. Phys., 2016, DOI: 10.1039/ c5cp05507e.

Since the publication of the original article, several matters that require clarification have been brought to our attention. In this correction article, we have provided further details on the characterisation of 9,10-bis(phenylethynyl)anthrancene (BPEA) and cited two other references to single-component-based multifunctional systems. ${ }^{1,2}$

Regarding the UV-vis absorption spectra shown in Fig. S2(b), these were recorded on BPEA powder dissolved in $\mathrm{CH}_{2} \mathrm{Cl}_{2}$ solution and nanosheets deposited on quartz. The spectrum of BPEA solution shows two peaks in the range of 350-470 nm, corresponding to an electronic $\pi-\pi^{*}$ transition. Compared with the monomeric species, the nanosheets exhibit a red-shift in the absorption peak and broadening of the spectrum. This can be attributed to the J-type aggregate of BPEA molecules in the nanosheets. A transition to the upper state in parallel aggregates has parallel transition moments. In this case, the shift is to lower energies of absorption, as described in the literature. ${ }^{3} \mathrm{~A}$ similar phenomenon was also reported in the literature. ${ }^{4}$

Although triple bonds in phenyleneethynylene are known to rotate freely (because of the very low rotational energy barrier), in this case, the XRD pattern reveals that the BPEA nanosheets can be indexed as monoclinic lattice (i.e., $\alpha$-phase), in which the BPEA molecule has a nearly planar structure (Fig. 1).

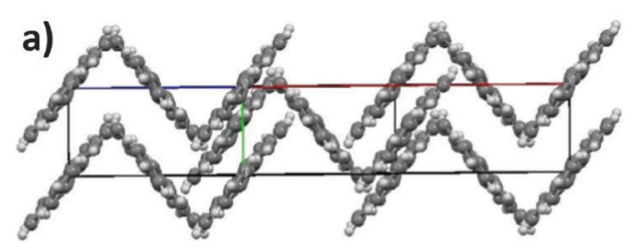

b)

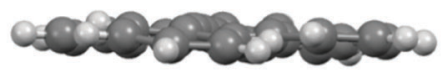

c)

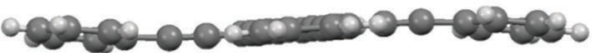

Fig. 1 (a) Simulated model of $\alpha$-phase BPEA. Molecular structures viewed along the long axis (b) and along the short axis (c).

In addition, it can be seen in Fig. 1(d) in the published article that the simulated XRD pattern of the single crystal of BPEA is not identical to the powder XRD of the nanosheets. Only six intense diffraction peaks are observed in the XRD of the nanosheets compared with the simulated pattern. Possibly this can be attributed to preferential growth of the nanosheets. The diffraction peaks in the XRD pattern of the BPEA nanosheets can be well indexed to the simulated pattern (Fig. 2).

\footnotetext{
${ }^{a}$ College of Chemistry and Environmental Engineering, Changchun University of Science and Technology, 130022 Changchun, China. E-mail: yjh@cust.edu.cn

${ }^{b}$ Suzhou Institute of Nano-tech and Nano-bionics, Chinese Academy of Sciences, 215123 Suzhou, P. R. China. E-mail: gbpan2008@sinano.ac.cn
} 


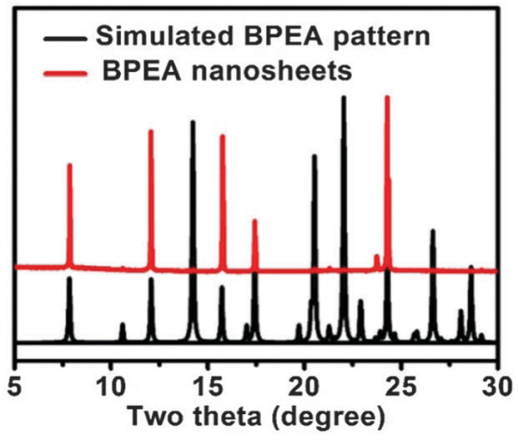

Fig. 2 XRD pattern of nanosheets and simulated pattern from the single crystal data.

We also did not include fluorescence spectra in the published article. Polarized photoluminescence spectra taken at different polarization angles are presented in Fig. 3. These were recorded on a single BPEA nanosheet deposited on a Si substrate with a focused laser $(405 \mathrm{~nm})$.

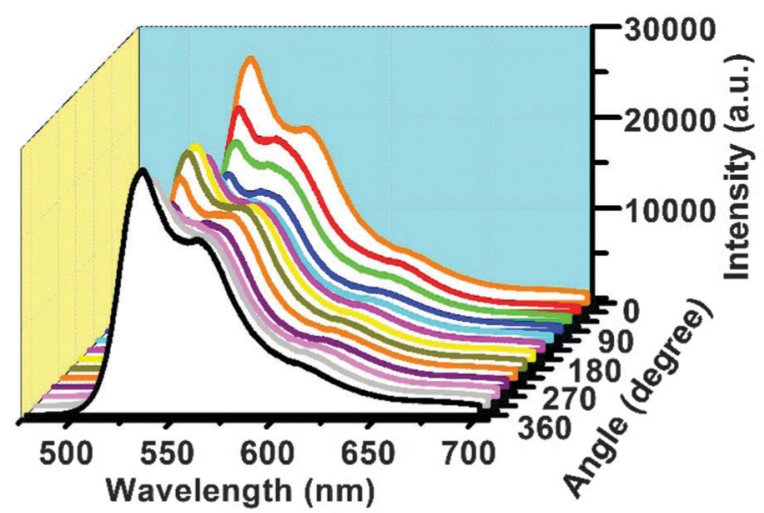

Fig. 3 Polarized PL spectra of BPEA nanosheet taken at different polarization angles.

Finally, we omitted to include two other examples of single-component-based multifunctional systems in the published article, and these are now cited here as ref. 1 and 2.

\section{References}

1 J. L. Chen, S. Q. Ma, J. B. Zhang, B. Li, B. Xu and W. J. Tian, ACS Photonics, 2015, 2, 313-318.

2 Q. Liao, H. H. Zhang, W. G. Zhu, K. Hu and H. B. Fu, J. Mater. Chem. C, 2014, 2, 9695-9700.

3 A. Eisfeld and J. S. Briggs, Chem. Phys., 2006, 324, 376-384.

4 Y. S. Zhao, J. J. Xu, A. D. Peng, H. B. Fu, Y. Ma, L. Jiang and J. N. Yao, Angew. Chem., Int. Ed., 2008, 47, 7301-7305.

The Royal Society of Chemistry apologises for these errors and any consequent inconvenience to authors and readers. 